\title{
SERONEGATIVE NECROTIZING MYOPATHY IN THE PRESENCE OF CORONAVIRUS INFECTION
}

\author{
Tatiana Melo Fernandes ${ }^{1, \star}$, Mariana D’Oliveira Bulhões da Costa ${ }^{1}$, Franciele Cristina Ferreira Pereira ${ }^{1}$, Walter Carlos de Oliveira \\ Bohrer ${ }^{1}$, Bartira Souza Melo ${ }^{1}$, Carlos de Oliveira Nascimento ${ }^{1}$, Maressa Barbosa Beloni Lirio ${ }^{1}$
}

1.Hospital Naval Marcílio Dias, Rio de Janeiro (RJ), Brazil.

*Corresponding author: tatianamelofernandes@gmail.com

\section{BACKGROUND}

Since February 2020, the COVID-19 pandemic has been reported with severe cases of flu-like illness whose symptoms were not restricted to the airways. Reports and studies have shown evidence of neurological involvement with reduced level of consciousness, seizures, cognitive and neuropsychiatric disorders, in addition to vascular involvement and muscle damage. Autoimmune necrotizing myositis can have acute or subacute symptoms, with severe muscle weakness and elevation of the creatine phosphokinase enzyme, which may occur after viral infections, collagenosis, use of statins or association with cancer. Cases of COVID-19 infection being a triggering factor for myositis are rare.

\section{CASE REPORT}

Female, 22 years old, no comorbidities, admitted in the hospital in February 2021, with flaccid tetraparesis and increased muscle enzymes. She presented strength grade 0 in the lower limbs, grade 1 in the right upper limb and grade 2 in the left upper limb. Electroneuromyography revealed myopathic potentials and signs of membrane instability. The patient reported the first dose of COVID-19 vaccine (Oxford) in late January, when she already had noninflammatory diarrhea, abdominal pain, myalgia and lowgrade fever. Magnetic resonance imaging of the thighs and arms showed muscle edema in the pelvic and scapular girdle muscles. Echocardiogram was normal. Esophageal transit scintigraphy showed slowed transit. Eye exam with yellowish lesions suggestive of multifocal retinitis associated with microhemorrhages. Laboratory tests with RF, anti-CCP, anti-RO, anti-La, anti-SM, anti-RNP, anti-DNA, anti-mitochondria, anti-Jo-1, anti-Mi-2, anti-SRP, anti-HMG-CoA were negative. Positive ANA with low titer 1:80 dense speckled nuclear pattern. The RT-PCR of coronavirus infection was positive in the oral and nasopharyngeal samples. A muscle biopsy of the vastus lateralis was performed, which showed extensive fiber necrosis and little inflammatory infiltrate. The patient was submitted to pulse therapy with $1 \mathrm{~g}$ methylprednisolone for five consecutive days without therapeutic response. The team also combined the above-mentioned therapy with venous gamma globulin at a dose of $27 \mathrm{~g} / \mathrm{day}$ for 5 days. She presented a good clinical and laboratory response and opted for the start of oral azathioprine and prednisone. After 4 months, she is asymptomatic with normal muscle enzymes and maintaining daily physical activity.

\section{CONCLUSION}

Coronavirus infection can have different forms of presentation, especially in young patients. It triggers autoimmune rheumatologic disease or mimic similar conditions, such as immune-mediated necrotizing myositis. Therefore, in times of pandemic, COVID-19 should always be excluded from clinical investigation.

\section{KEYWORDS}

Necrotizing myopathy, Coronavirus infection, Myopathy. 